\title{
M.A.R.S - Mechanized Air Refilling System
}

\author{
P.Omprakash ${ }^{1}$, T.Senthil Kumar ${ }^{2}$ \\ ${ }^{1}$ Assistant Professor \\ ${ }^{1,2}$ Velammal College of Engineering and Technology, Madurai
}

\begin{abstract}
:
Every section of an automobile is getting automated except one-Tires. To inflate a tire, the driver has to go to a gas station or he has to attach a pump manually. Both these involve human labor. This project is aimed at removing such unwanted strain and save time. The system has a dedicated unit for filling air whenever required. A control unit which is a pic microcontroller is the brain of the system. When the pressure level is below the threshold value chosen by the driver, the system displays it. The driver then may choose to refill the air automatically. A compact pump does the job. Another problem in tires is a puncture. A sudden puncture can cause the driver to lose control, culminating in an accident. The project's minor objective revolves around this. During a puncture the air pressure reduces suddenly. This reduction in pressure over a time limit is identified as a puncture. It warns the driver of a puncture and saves life. The other way of detecting a puncture is by calculating the ON time of the pump. If the pump is in ON state for a long time and there is no improvement in the pressure level it is recognized as a puncture. It also saves a lot of money by giving more longevity to the tire and better mileage. As the tire is filled with optimum air, the friction between the tire and the road is maintained properly. Henceforth, the tire is not damaged much and the fuel consumption is reduced. The project is destined to be a life and money saver.
\end{abstract}

\section{Introduction:}

Automobiles have become an important and reliable companion of humans. The usage of the automobiles is increasing in a rapid manner. The various Automobile industry are now competing each other to win the hearts of humans. In order to do so, the companies are improving the safety systems in automobiles. The more reliable, the more successful the car becomes.

GM has devised a system which helps the owner to find his car in a parking lot, send emergency messages with location of the car during an accident. This is a lower end safety system. Taking to higher level, Volvo has devised a system which brakes automatically when it senses an obstacle on its path. Bulletproof cars, ABS systems, GPS tracking, Preventing drunken drive \& sleep over wheels; Air bags are some other safety systems in cars. Even though there are many security systems, small factors such as under inflation and punctures make human life misearable. The safety systems are not concentrated over the wheels. In Texas August 2008, a bus accident occurred due to tire puncture. 17 were killed and 39 were injured. This incident set an alarm among the various automobile companies to concentrate on the tires. 


\section{Existing Systems:}

The following three are the existing systems that focus on tires:

- CTIS- Central Tire Inflation System

- TIREMAAX

- AIRGO

All the above mentioned systems are not available for commercial cars and they are not a dedicated system.

The Central Tire Inflation System is used by the military. As said the system is not a dedicated one. Air brake system and CTIS system use the air from the same provision because which a problem occurs. You cannot use both the system at the same time. Doing so may result in Accidents. Below shown is a military truck that has toppled due to the above problem.

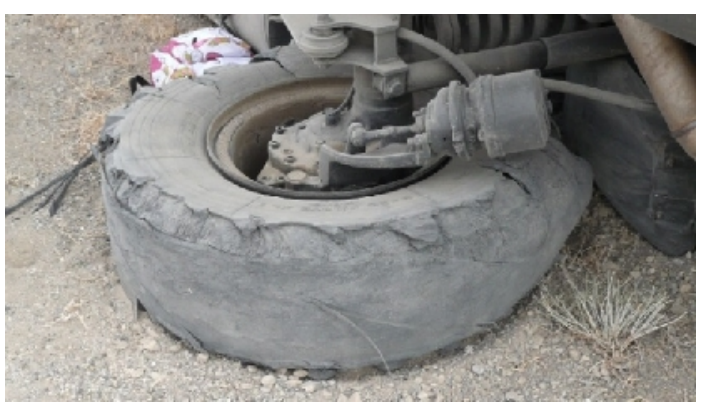

Fig 1- Accident due to CTIS

So we have to design a dedicated system only for refilling purpose. The existing system of air refilling requires manual labor. Physical connection of tire and the pump must be done every time, this is time consuming. Other problems are, the tire must be filled with optimum pressure level. There must be no under inflation or over inflation. Large tires of trucks require special arrangements. This is to avoid injuries if the tire explodes due to over inflation.

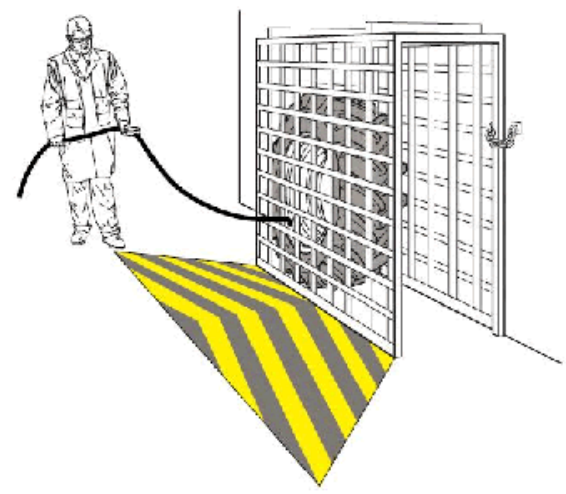

Figure 2- Protected pumping 


\section{Proposed Work:}

\subsection{What is M.A.R.S and why?}

Automation and automobiles go hand in hand. Every section of an automobile is getting automated except one- Tires. To inflate a tire, the driver has to go to a gas station or he has to attach a pump manually. Both these involve human labor. This project is aimed at removing such unwanted strain and save time.

Another problem in tires is a puncture. A sudden puncture can cause the driver to lose control, culminating in an accident. The project's minor objective revolves around this. It warns the driver of a puncture and saves life. It also saves a lot of money by giving more longevity to the tire and better mileage. As the tire is filled with optimum air, the friction between the tire and the road is maintained properly. Henceforth, the tire is not damaged much and the fuel consumption is reduced. The project is destined to be a life and money saver.

\subsection{Description:}

The system can be divided into three:

- Control

- Pumping and

- Display unit

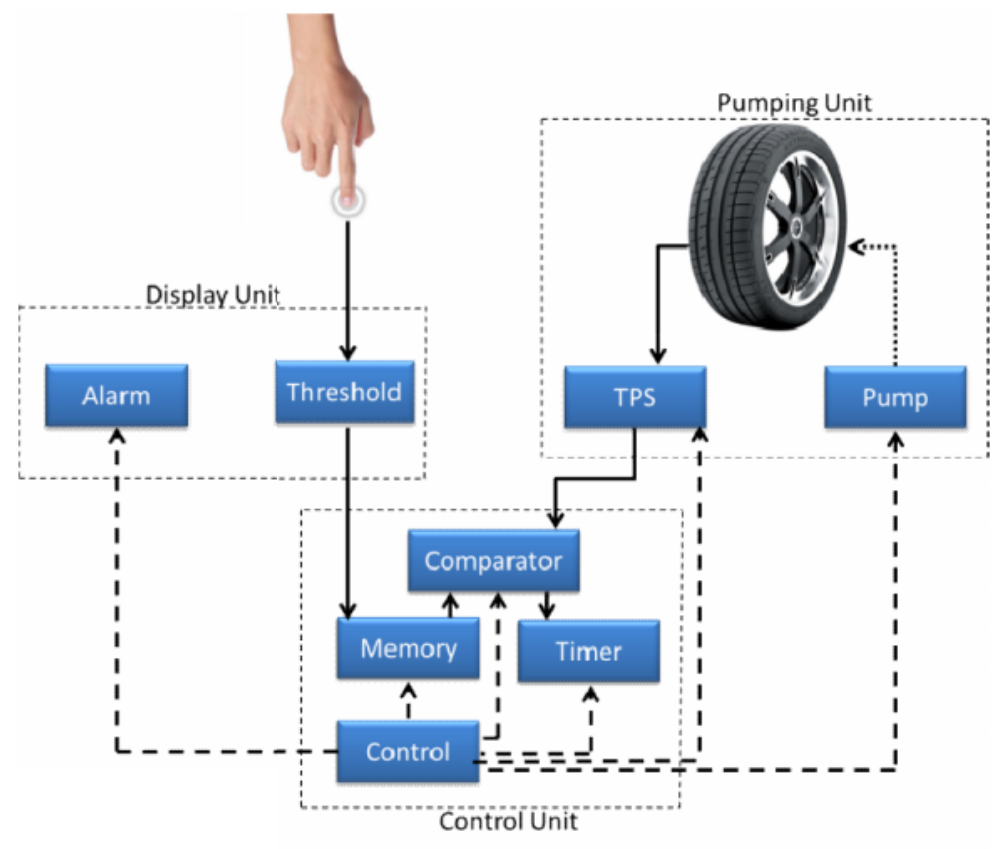

Figure 3- Block Diagram 


\subsubsection{Pumping unit:}

It comprises of the compact pump, hose, specially designed wheel alloy and the tire. The wheel is designed to serve the purpose of pumping and wheel rotation with minimal air leakage. The pump is fitted to the car either magnetically or screws. Magnetic fixing is to avoid the pump from falling at any cost. The pump will be fitted near the wheel, parallel to its axle. Each wheel will get a dedicated pump.

The pump needs just $12 \mathrm{v}$ to operate. So a power supply from the car battery is more than enough for the proper functioning of the pump. The hose is made of a strong material to survive the rough conditions.

Next part in this unit is the wheel alloy. The alloy is modified to suit this project. This project involves the connection of the pump and the tire's valve permanently, even during the motion of the car. In this case, the wheel's rotation must not damage the pump connection at any cost. So we go for a special design which facilitates the motion of the tire without damaging the connection. The design provides an airtight connection in the center of the wheel. This allows free motion. The valve used is a one way valve, to prevent the air from escaping through the same.

The tire used may be a tube or a tubeless one, but the system remains the same. The connection between valve and hose is a permanent one. This doesn'tmean that it cannot be removed at time of a puncture. Permanent in the sense, even during the motion of the car. It can be removed by the driver while changing a tire. The above parts comprise a single section for one wheel. So four sections totally make up the entire pumping unit.

\subsubsection{Display unit:}

This is not only a unit which gives information but also gets some data from the driver. A touch system will be well and good. On the first installation of the unit, the driver chooses his desired pressure level. This will be stored in the system's memory as the threshold value. This value can be changed by the driver depending upon the load of the vehicle. The optimum level is about 35-40 psi. When the driver starts the car, the system is switched ON and shows two options- Manual or Auto mode. As the name suggests, one is fully automatic while the other gets authentication from the driver every time it has to perform an action. For convenience let's consider that the user has selected the auto mode. The system measures the pressure level in each tire and displays it for each wheel. The driver can choose either all the wheels or one that requires to be filled. The tire is filled to the required level and the unit displays the completion of the process.

The other information that is displayed is about the puncture. Both the alarm and display unit combines to warn the driver about the predicted puncture.

\subsubsection{Control unit:}

This is the brain behind the system. It takes major decisions and coordinates the entire system. The inputs given to the system are from the display unit and TPS. Output is given to the display unit and pump. The following actions are taken by the control unit in order:

\section{Air refilling:}

- Pressure measurement

- Display the pressure to the driver

- Get the input and signaling the corresponding pump to start 
- Checks for the threshold attainment

- Signals back to the driver

\section{Puncture predictor-}

As said before the unit also warns about a puncture. The control unit is solely responsible for this act. It checks the pressure level say for every 30 seconds. Let us consider this case. The threshold level is chosen as 40 . The control unit measure and cross checks for every 30 seconds. If the pressure varies or is between 35-40psi (approximately) during the motion of the car, it may be due to the frictional heat. In case of a puncture, pressure drops slowly. The control unit will be comparing the pressure levels regularly. When the pressure becomes below, the control unit changes the threshold value. It reduces it about 5 psi. the unit compares the pressure level again. This is done for 5 loops. If the pressure level is below the threshold level even after the $5^{\text {th }}$ loop, the control unit recognizes this as a puncture and warns the driver through the display and alarm system. The alarm will be switched off automatically once the car is stopped.

The best time for checking air is before the start of a journey. This is because during or after a journey, the tire gets heated during friction. Due to this the pressure level will vary. Taking measurements as such time is not valid. Doing it before a journey is the optimum condition for pressure measurement. Research shows the same.

\section{Simulation:}

Its always better to check a result before fabrication of the device. The following results show the simulated verification of the idea designed.

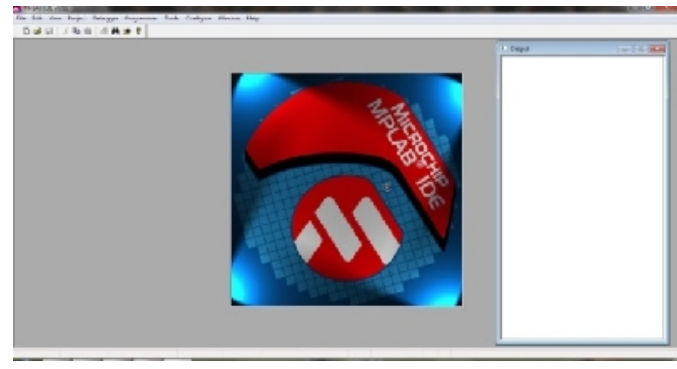

Figure 4 - MP Lab

The program for the PIC 16f877A was written using MP Lab version 8.

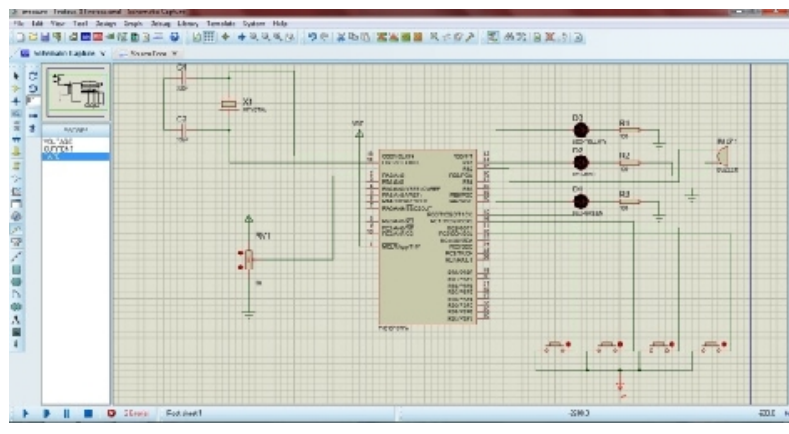

Figure 5- Proteus 
The simulation of my proposed idea was done in proteus software. Fig A shows the circuit diagram of my prototype.

Instead of a pressure sensor in a tire, a variable resistance is given as the input. There are three status LEDs - Green, Yellow and Red. The Green Led is switched on whent the existing pressure is above the threshold pressure.

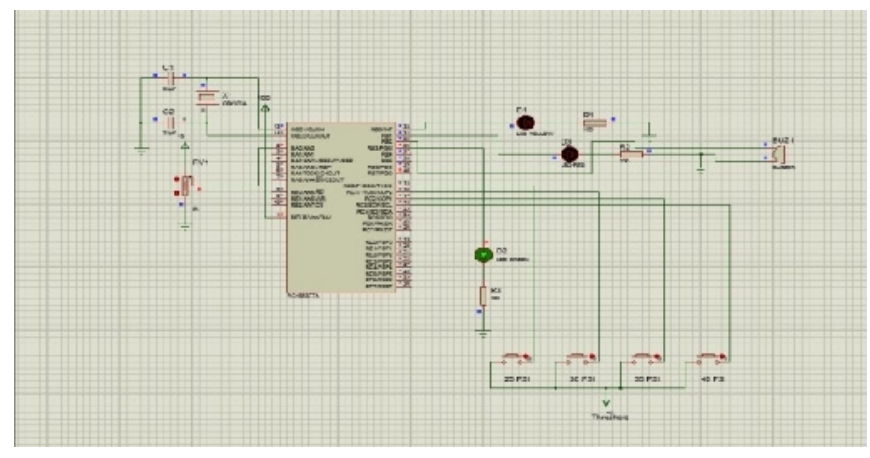

Figure6 - Normal Pressure - Green Light

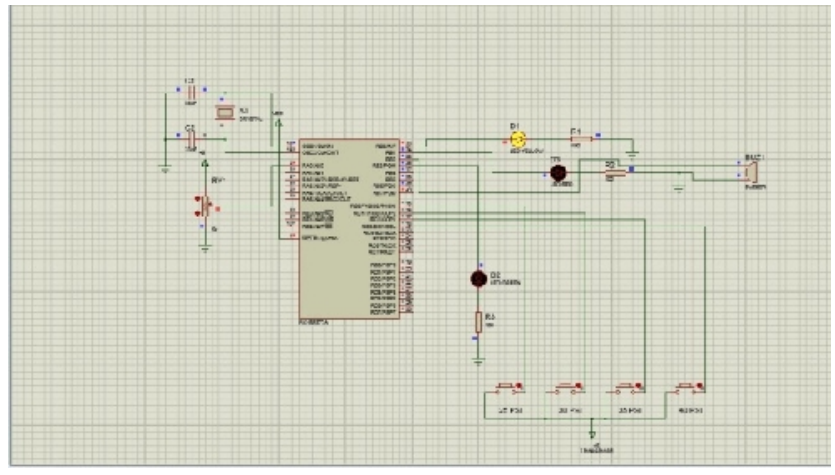

Figure 7 - Below thershold - Yellow Light

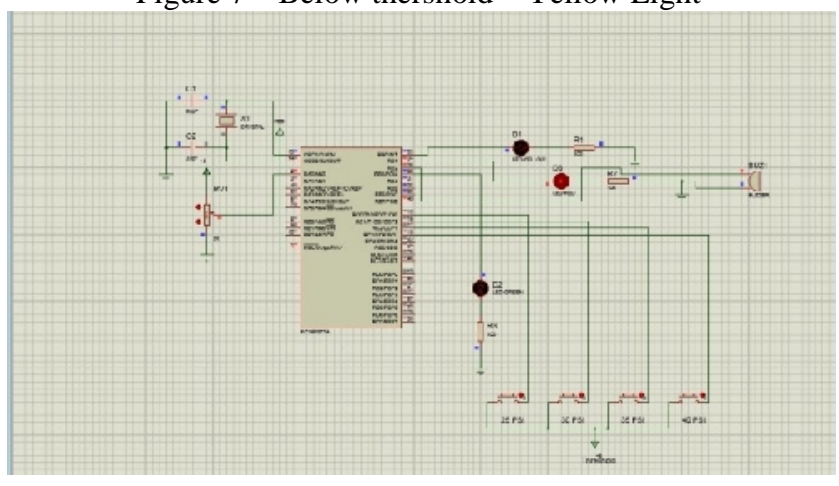

Figure 8- Puncture - Red Light and Buzzer

When the pressure drops below the threshold, Yellow Led is switched on and Green is in the low state. Red Led is switched on under two conditions. The conditions are - If the yellow Led is ON for a period of 10 s or when the pressure has a sheer drop below the threshold value. 
The first condition is analogus to the condition of the pump in $\mathrm{ON}$ condition for a long time. If the Pump is ON and there is no improvement in the pressure level, it is obviously a puncture. As soon as a puncture is identified, the Red Led and the buzzer is switched ON. This is the Alarm system of M.A.R.S.

\section{Results and Discussion:}

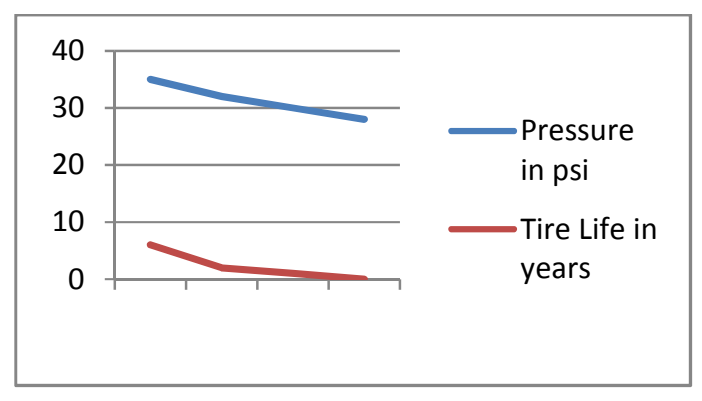

Figure 9- Before System

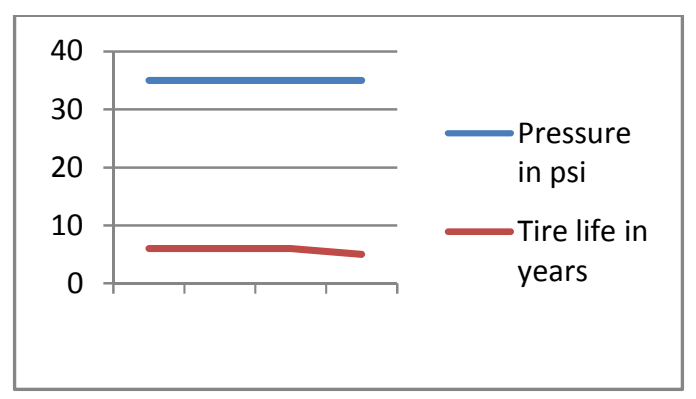

Figure 10- After System

The two graphs show a comparison between the pressure level in tire and the life time of the tire before and after using the system. The manufactures say that the maximum life time of a tire is mostly around 6 years.

Figure 1- Before using the system, the pressure level is not maintained properly. Due to this improper maintenance of the tire, the life time of the tire falls rapidly. This causes the early replacement of tire. The under inflation also causes more wear and tear of the tire. This under inflation allows punctures to occur easily.

Fig 2- After using the system, the pressure level is maintained properly. The tire is filled with optimum air. Thus the life time of the tire ismaintained properly. As it is filled with optimal air the probability of puncture is greatly reduced

\section{Conclusion:}

The analysis showed clearly that my system improved the life time of the tire and also provided a smooth ride. It also increased the fuel efficiency. The prototype was able to tackle various conditions. It also proved vital in warning about the puncture. My future implementation is to device a more compact version of my prototype. 
International Journal of Information Sciences and Techniques (IJIST) Vol.4, No.3, May 2014

\section{References:}

[1] Device for the automatic actuation of a tire filling apparatus for a motor vehicle-Heinz Christely and Gerhard Skoff (US20030230342 A1)

[2] Dynamically-Self-Inflating Tire System- Michael Alexander, Anthony Brieschke, Jonathan Quijano, and Lau Yip

[3] Apparatus for the automatic filling of motor vehicle tires- Skoff, Gerhard

[4] Automatic tire inflation system-Ellison Michael J, Goodwell, Fred L (US4418737A)

[5] Automatic tire pressure control system for a vehicle- Rober A.Genna

[6] Automatic tire inflation system retrofitting kit- Ellison Michael J, Goodwell, Fred L 